\title{
Two Cases of Crossed Cerebellar Diaschisis with or without Thalamic Lesion on Brain MRI in Status Epilepticus
}

\section{Case Report}

Journal of Epilepsy Research pISSN 2233-6249 / elSSN 2233-6257

Received September 12, 2014

Accepted October 28, 2014

Corresponding author: Kwang Ki Kim Department of Neurology, Dongguk

University Ilsan Hospital, 27

Dongguk-ro, Ilsandong-gu, Goyang

410-773, Korea

Tel. +82-31-961-7215

Fax. +82-31-961-7277

E-mail; neukim@duih.org

\author{
Hee Seung Ahn, Kwang Ki Kim \\ Department of Neurology, Dongguk University Ilsan Hospital, Dongguk University College of Medicine, Goyang, \\ Korea
}

\begin{abstract}
We report two cases of status epilepticus who showed crossed cerebellar diaschisis (CCD) with or without pulvinar high signal intensity (HSI) on brain magnetic resonance imaging (MRI). The first patient had CCD with pulvinar HSI on MRI and periodic lateralized epileptiform discharges (PLEDs) on electroencephalography. MRI of the second patient revealed CCD without pulvinar HSI. And electroencephalography (EEG) of this patient did not show PLEDs. The authors suggest that the pulvinar lesion might be related to the generation of PLEDs in Status epilepticus. (2014;4:74-77)
\end{abstract}

Key words: Status epilepticus, Pulvinar, Crossed cerebellar diaschisis, Periodic lateralized epileptiform discharges

\section{Introduction}

Brain magnetic resonance imaging (MRI) is an essential examination for finding brain lesions in epileptic diseases. Except for lesions causing status epilepticus, findings of brain MRI characteristic to status epilepticus patients are not observed commonly, but with the advance of brain imaging technology, abnormal signals in various regions have been reported. ${ }^{1}$ Particularly in status epilepticus patients, reports of abnormal findings in diffusion weighted image (DWI) and fluid attenuated inversion recovery (FLAIR) images are increasing gradually. Thus, the author reports imaging findings and electroencephalography (EEG) findings of a status epilepticus patient in whom crossed cerebellar diaschisis and thalamic lesions were observed at the same time.

\section{Cases}

\section{Case 1}

A 50-year-old male patient visited the hospital for decreased consciousness accompanied by generalized tonic-clonic seizure. In his past history, the patient has been an alcoholic drinking a bottle of soju 5 or more days in a week for several years, and generalized tonic-clonic seizure lasting for about 5 minutes occurred 3 months ago and he was examined at another hospital for the condition but the patient refused to get tests at that time. The patient was found by a guardian while his four limbs were in a tonic-clonic seizure, but as the symptom continued for about an hour without stopping he was brought to the emergency room. On admission to the emergency room, his vital signs were blood pressure 160/90 mmHg, pulse rate 178 times/min, respiratory rate 38 times/min, and body temperature $39.0^{\circ} \mathrm{C}$. Blood test results were total bilirubin $1.7 \mathrm{mg} / \mathrm{dL}$, prothrombin time (PT)/international Normaized Ratio (INR) 1.38, aspartate aminitransferase (AST) 554 IU/L, alanine transaminase (ALT) 104 IU/L, and lactate dehydrogenase (LDH) $540 \mathrm{IU} / \mathrm{L}$. The state of consciousness was semi-coma, and generalized tonic-clonic seizure continued. The seizure stopped when $4 \mathrm{mg}$ of lorazepam was infused intravenously, but it recurred in 3 minutes and therefore additional 3 $\mathrm{mg}$ of midazolam was administered intravenously. The symptom was diagnosed as status epilepticus, and soon 1,200 mg of fosphenytoin was administered. Later, $350 \mathrm{mg}$ of fosphenytoin and 1,000 mg of levetiracetam were administered at a maintenance dose. In DWI, high signal intensity was observed in the cortices of right posterior frontal lobe, parietal lobe, temporal lobe and occipital lobe, right thalamus, right hippocampus, and left cerebellum (Fig. 1A), in apparent diffusion coefficient, high signal or isosignal intensity was observed in the same regions (Fig. 1B), and in FLAIR, high signal intensity was observed in the same regions (Fig. 1C). The results of spinal fluid examination were normal, and in EEG, periodic lateralized epileptiform discharges (PLEDs) were confirmed in the entire right hemisphere (Fig. 1F). The patient's consciousness maintained a hyp- 

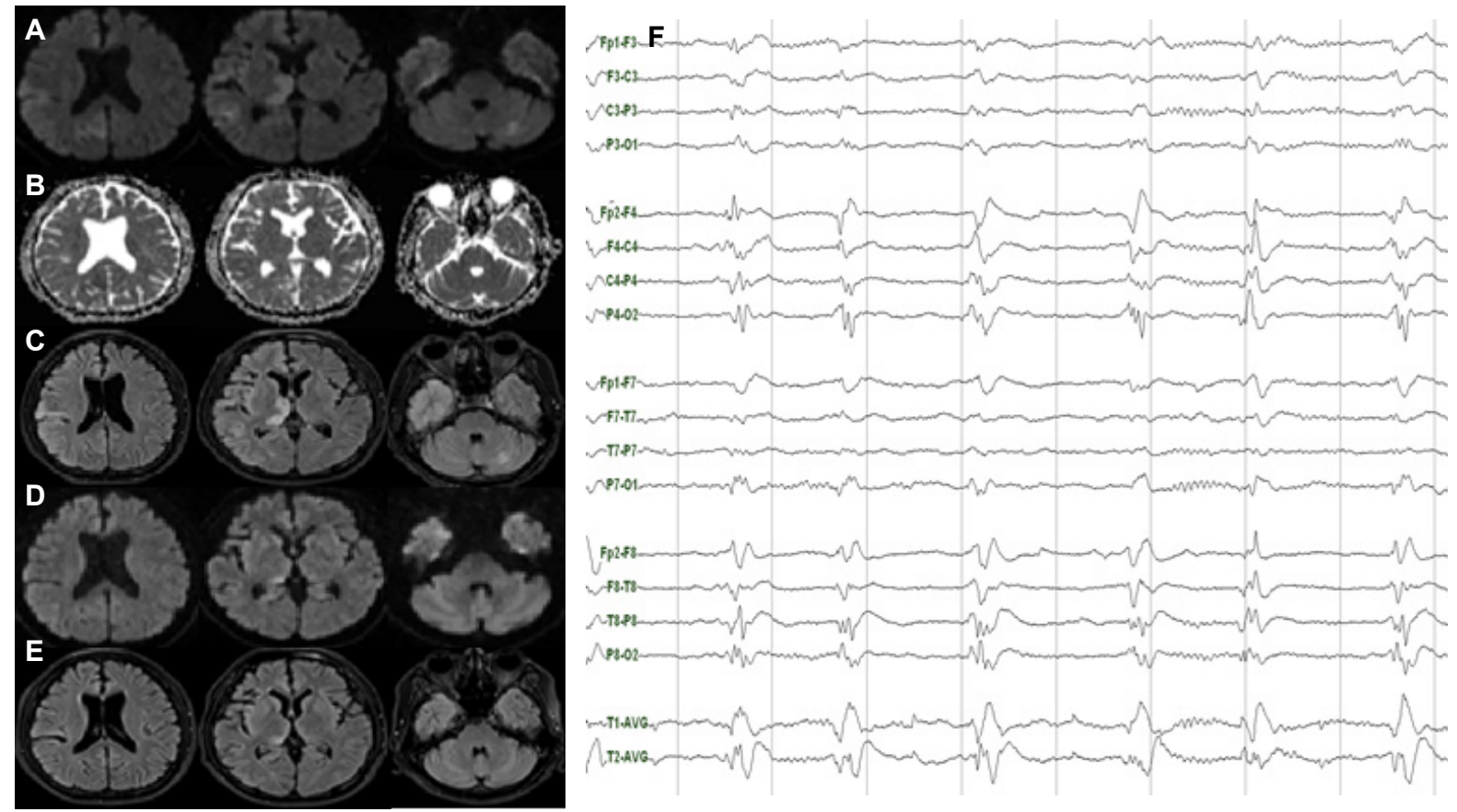

Figure 1. Brain MRI and EEG of the patient 1. Initial DWI showed multiple areas of high signal intensity in the right fronto-parieto-occipital cortex, right pulvinar and left cerebellar hemisphere (A). ADC showed iso or low signal intensity in corresponding areas (B). FLAIR revealed high signal intensity in corresponding areas (C). Follow up DWI (D) and FLAIR (E) images (after 3 weeks) showed decreased signal intensity in previously affected areas. Initial EEG showed periodic lateralized epileptiform discharges (PLEDs) over the right hemisphere (F). MRI, magnetic resonance imaging; EEG, electroencephalography; DW, diffusion weighted image; ADC, apparent diffusion coefficient; FLAIR, fluid attenuated inversion recovery.

noleptic state, and in 24-hour EEG conducted for 4 days, PLEDs were observed continuously in the right hemisphere. He recovered his consciousness in 7 days of admission, and follow-up DWI and FLAIR after 3 weeks showed reduced signal changes in the right frontal lobe, parietal lobe, temporal lobe, occipital lobe, and right thalamus, and the disappearance of abnormal findings that had been observed in the left cerebellum (Fig. 1D, 1E).

\section{Case 2}

A 38-year-old woman visited the hospital for the behavior of smacking her lips and intermittent memory loss since a month ago. In her past history, she was diagnosed with parietal lobe epilepsy 8 years ago, and had been taking $100 \mathrm{mg}$ of lamotrigine as antiepileptic twice a day. While staying in the hospital, she suddenly had generalized seizure with showing the twitching of the left face, left gaze preference, and the stiffness of the left arm and leg, so $4 \mathrm{mg}$ of lorazepam was infused intravenously. Then, she showed a consciousness of hypnoleptic state, and after about 10 minutes, local seizure recurred and additionally $3 \mathrm{mg}$ of midazolam was administered intravenously. As the seizure did not stop for over 30 minutes, it was diagnosed as status epilepticus and 1,500 mg of fosphenytoin was administered, and then $200 \mathrm{mg}$ of lamotrigine, 2,000 $\mathrm{mg}$ of levetiracetam, and $900 \mathrm{mg}$ of oxcarbazepin were administered at a maintenance dose. In DWI, high signal intensity was observed in the right parietal lobe, part of the temporal lobe, and the insular cortex (Fig. 2A), and in apparent diffusion coefficient (ADC) as well, isosignal or low signal intensity was observed in the same regions (Fig. 2B), and in FLAIR, high signal intensity was observed in the same regions and in the left cerebellum (Fig. 2C). During routine EEG were observed accidently seizure symptoms such as paresthesia of the left face and twitching of the face, and in EEG around that time was observed slow wave with irregular rhythm of about 4-5 Hz. in the right temporal region (Fig. 2F). On the following day, clear consciousness was recovered, and the results of spinal fluid examination were normal. In follow-up DWI and FLAIR after 2 weeks, the lesions showing abnormalities disappeared (Fig. 2D, 2E).

\section{Discussion}

Brain lesions that may be found in status epilepticus patients are 

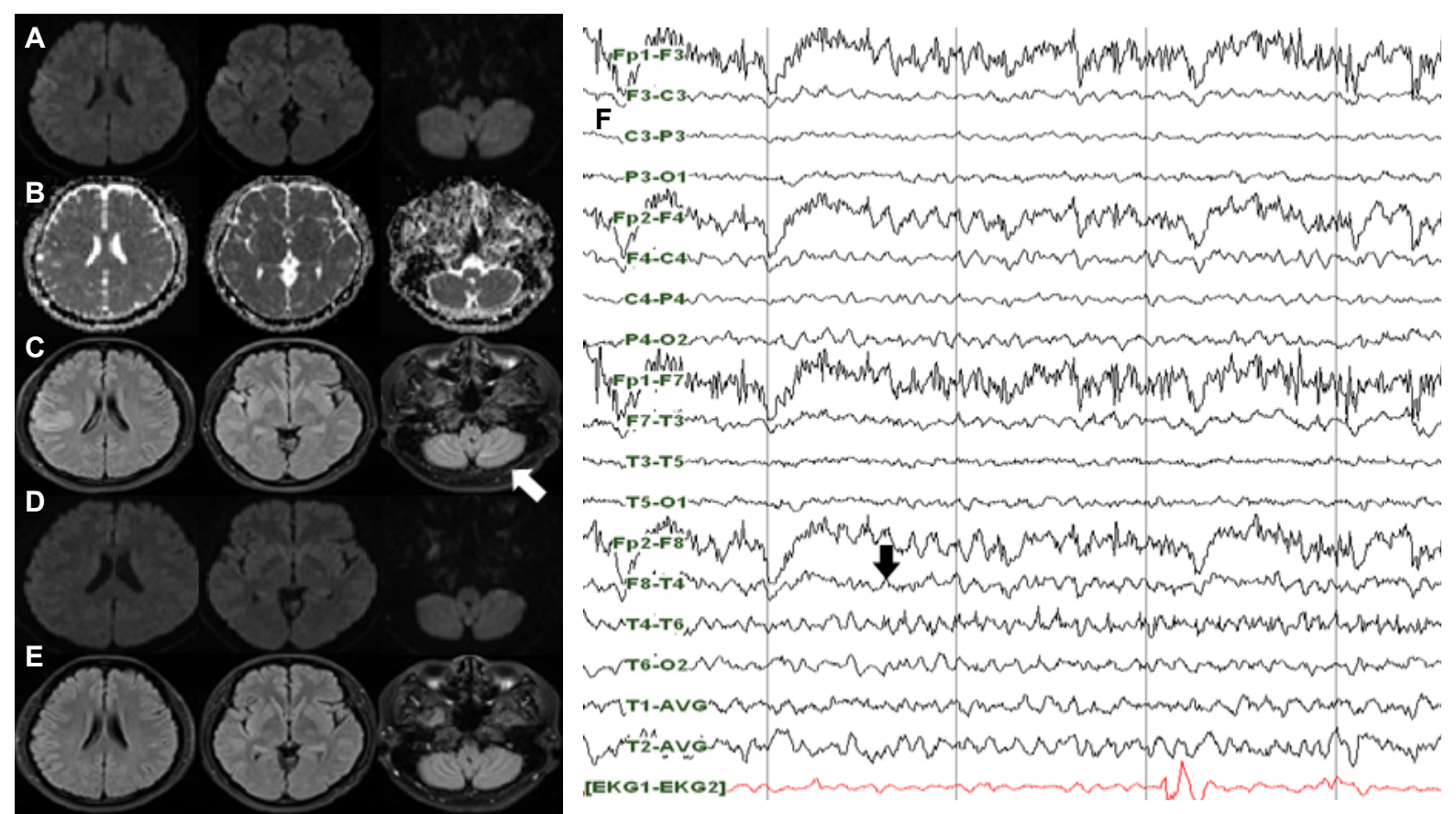

Figure 2. Brain MRI and EEG of the patient 2. Initial DWI showed high signal intensity in the right parieto-temporal cortex and insular cortex (A). $A D C$ showed iso or low signal intensity in corresponding areas (B). FLAIR images revealed high signal intensity in corresponding areas and left cerebellar hemisphere (white arrow) (C). In follow up DWI (D) and FLAIR (E) images (after 2 weeks), these abnormal findings are disappeared. Ictal EEG showed a regional slow activity with irregular rhythm on right temporal area without PLEDs (F). MRI, magnetic resonance imaging; EEG, electroencephalography; DW, diffusion weighted image; ADC, apparent diffusion coefficient; FLAIR, fluid attenuated inversion recovery; PLEDs, periodic lateralized epileptiform discharges.

crossed cerebellar diaschisis, signal enhancement in pulvinar, caudate nucleus, globus pallidus, putamen, and splenium of corpus callosum, the dilation of cortical vessels on the affected side, etc. ${ }^{1}$ These brain lesions in status epilepticus patients are usually reversible, but sometimes they can be irreversible and cause a permanent damage. Crossed cerebellar diaschisis is believed to take place as a disorder in the afferent corticopontocerebellar pathway is caused by a supratentorial lesion and, as a result, blood flow and oxygen decrease in the cerebellar hemisphere of the opposite side. ${ }^{2}$ In a status epilepticus patient, glutamate is discharged continuously and it induces desensitization of gamma-aminobutyric acid type $A\left(G A B A_{A}\right)$ and, consequently, the excitement of neurons continues and cerebral metabolism and oxygen demand increase. ${ }^{3}$ It is believed that, as a result, regions on the corticopontocerebellar pathway that fail to meet the excessively increased cerebral metabolic demand are damaged.

While a temporal lobe epilepsy patient is in seizure, the thalamus interacts with the temporal lobe, and particularly it has been reported that the pulvinar situated on the dorsomedial aspect of the thalamus plays an important role in transduction from the cortex where the seizure takes place to other regions. ${ }^{4}$ This suggests that the corticothalamic pathway is activated during the seizure, and the thalamus forms a signaling network with the cerebral cortex of other regions and, in this way, plays an important role in causing generalized seizure. It was also reported that because there may be a network inducing epilepsy formed already between the thalamus and other cortex in a temporal lobe epilepsy patient, even if temporal lobectomy is performed the prognosis may not be favorable. ${ }^{4}$ According to some reports, an extensive region of the thalamus is activated at the early stage of status epilepticus, and pulvinar lesions are observed at the later stage of status epilepticus and are involved in the suppression of signals related to seizure. ${ }^{5}$ What is more, it was reported that the signal intensity of the pulvinar in brain MRI was different according to the severity of seizure that occurred in the cortex. ${ }^{6}$ In this way, the thalamus is believed to play an important role as a pathway in mediating seizure-related signals among the cerebral cortex.

In a study by Katramados with 36 partial status epilepticus patients, 11 (31\%) showed thalamic lesions, and PLEDs were observed in all of the 11 patients. In addition, PLEDs were observed in 17 $(68 \%)$ of the 25 patients not showing thalamic lesions. ${ }^{5}$ That study 
reported that there was no statistical association between PLEDs and thalamic lesions, and rather thalamic lesions were associated with brain lesions in posterior parts such as occipital lobe, parietal lobe, and temporal lobe and with the continuity of seizure. Also, it reported that among the thalamic nuclei the pulvinar appeared to be the structure playing the most important role in signal transduction. In DWI of these cases, cortical lesions were more extensive in patient 1 who had lesions in the pulvinar than in patient 2, and the seizure in EEG lasted longer in patient 1. These findings were similar to previous reports.

It has not been clarified whether PLEDs, which were described for the first time in 1964, are caused by epileptic seizure or are secondary events caused by lesions. ${ }^{7}$ PLEDs have been reported in patients with stroke, brain tumor, epilepsy, herpes encephalitis, metabolic encephalopathy, etc., and particularly in connection to epilepsy, they are known to be related to clinical seizure and it is believed that they occur due to disconnection between deep intracerebral structures such as basal nuclei and thalamus and cerebral cortex. In the patient of our case as well, along with ipsilateral extensive cortical and thalamic lesions, PLEDs of $0.5-1 \mathrm{~Hz}$ were observed in the hemisphere of the same side in EEG. As the patient's consciousness decreased while PLEDs were observed, it was thought to suggest a state of non-convulsive seizure. Although previous studies failed to prove a statistical association between PLEDs and thalamic lesion, ${ }^{5}$ and there was a report in Korea that PLEDs were not observed in a non-convulsive status epilepticus patient who had thalamic lesions, ${ }^{8}$ it may be hard to exclude completely the association between the thalamus and PLEDs in status epilepticus in consideration that all of status epilepticus patients with thalamic lesions showed PLEDs in previous studies and in this case as well. ${ }^{5}$

Patient 1, who was a chronic alcoholic, can be classified into the category of subacute encephalopathy with seizure in alcoholics (SESA). SESA is a disease of a chronic alcoholic characterized by seizure along with local neurologic disorder, and PLEDs in EEG, and is known to cause status epilepticus. ${ }^{9}$ In patient 1 , local neurologic disorder was not found clearly but SESA was suspected in the patient because of the history of chronic alcoholism, elevation of liver enzyme levels in serologic test, and PLEDs in EEG. Patient 2, without a history of alcoholism, had shown right parietal lobe epilepsy, and as it was aggravated with the stopping of medication, the patient had shown continuous partial seizure and intermittent generalized seizure. The patient's seizure focus was believed to be the right parietal lobe showing high signal intensity in DWI, and crossed cerebellar diaschisis was observed, but lesions were not observed in the pulvinar and PLEDs were not observed in EEG.

This case report compared a patient having lesions in the cerebral cortex and pulvinar, crossed cerebellar diaschisis, and PLEDs with a status epilepticus patient who showed only lesions in the cerebral cortex and crossed cerebellar diaschisis with neither pulvinar lesions nor PLEDs observed. In a status epilepticus patient, crossed cerebellar diaschisis or signal enhancement in the thalamus may happen in brain MRI due to the overactivity or disorder of seizure-related cortex and pathway connected to them. Such brain imaging findings tend to appear more frequently in status epilepticus patients with uncontrollable or long-lasting seizure, and in particular thalamic lesions are believed to be related to the progression of seizure.

\section{References}

1. Huang YC, Weng HH, Tsai YT, et al. Periictal magnetic resonance imaging in status epilepticus. Epilepsy Res 2009;86:72-81.

2. Baron JC, Bousser MG, Comar D, Castaigne P. "Crossed cerebellar diaschisis" in human supratentorial brain infarction. Trans Am Neurol Assoc 1981;105:459-61.

3. Goodkin HP, Joshi S, Mtchedlishvili Z, Brar J, Kapur J. Subunit-specific trafficking of $\operatorname{GABA}(\mathrm{A})$ receptors during status epilepticus. I Neurosci 2008;28:2527-38.

4. Guye M, Regis J, Tamura M, et al. The role of corticothalamic coupling in human temporal lobe epilepsy. Brain 2006;129:1917-28.

5. Katramados AM, Burdette D, Patel SC, Schultz LR, Gaddam S, Mitsias PD. Periictal diffusion abnormalities of the thalamus in partial status epilepticus. Epilepsia 2009;50:265-75.

6. Sharma V SR. Thalamic neuropathology in the chronic pilocarpine and picrotoxin model of epilepsy. Indian J Exp Biol 1999;37:461-7.

7. Chatrian $G E$, Shaw $C M$, Leffman $H$. The significance of periodic lateralized epileptiform discharges in EEG: an electrographic, clinical and pathological study. Electroencephalogr Clin Neurophysiol 1964;17:17793.

8. Koo DL, Jeong HG, Nam HW. Thalamic hyperintensity on diffusionweighted MRI in a patient with nonconvulsive status epilepticus. Journal of Epilepsy Research 2013;3:32-4.

9. Niedermeyer E, Freund G, Krumholz A. Subacute encephalopathy with seizures in alcoholics: a clinical-electroencephalographic study. Clin Electroencephalogr 1981;12:113-29. 